\title{
Human-Machine Cooperative Grasping/Manipulating System Using Force-based Compliance Controller with Force Threshold
}

\author{
Naoki Motoi* Member, Ryogo Kubo** Member
}

(Manuscript received March 16, 2015, revised July 23, 2015)

\begin{abstract}
In recent years, there have been several studies on human-machine cooperative systems. These systems involve contact between humans and the environment. Therefore, it is necessary to create a safe system in order to avoid injuring humans and the environment. On the other hand, task realization is also important. From this viewpoint, a force-based compliance controller with a force threshold is proposed in this paper. This controller achieves both task realization and adaptation to the environment. This motion selection is conducted by comparing the force command and a force threshold. In addition, a human-machine cooperative grasping/manipulating system using the proposed controller is developed. This system can conduct a human-machine cooperative motion, which consists of an autonomous motion and a human-assisting motion. The priorities of the autonomous motion and the human-assisting motion are easily designed by changing the force thresholds. The validity of the proposed system is confirmed with experimental results.
\end{abstract}

Keywords: motion control, human-machine cooperative system, force controller, compliance controller

\section{Introduction}

Many industrial robots have been installed for use around the world. These robots have contributed to increases in productivity in manufacturing facilities. Technology development for these industrial robots has focused on disturbance suppression for high-accuracy motion. High-accuracy motion has mainly been achieved by position-based control. When the industrial robots are in contact with humans or the environment, they treat the contact force as a disturbance. Therefore, human operators could be injured or the environment damaged. In order to avoid this situation, industrial robots were only installed for work in structured environments and workers were prohibited from entering the movable area of the industrial robots.

In 2011, the International Organization for Standardization (ISO) modified ISO10218, which is the safety standard for industrial robots ${ }^{(1)}$. As a result, humans are now allowed to enter the movable area upon completion of a risk assessment. Due to this, several types of the human-machine cooperative systems are expected to be developed.

Figure 1 shows the concept of the human-machine cooperative system. For example, two workers grasp and manipulate the object as shown in Fig. 1(a). This grasping/manipulating motion by workers is one of the typical tasks in the manufacturing area. Figure 1(b) shows one of the human-machine cooperative systems. In this system, the grasping motion is autonomously controlled. The manipulating motion is assisted by the worker. Then, the number of workers is reduced.

\footnotetext{
* Graduate School of Maritime Sciences, Kobe University

5-1-1, Fukaeminami, Higashinada-ku, Kobe 658-0022, Japan

** Department of Electronics and Electrical Engineering, Keio University

3-14-1, Hiyoshi, Kouhoku-ku, Yokohama 223-8522, Japan
}

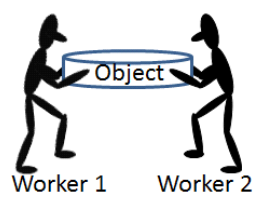

(a) Work by only workers

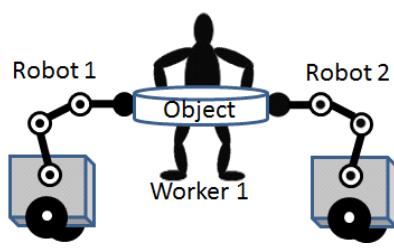

(b) Human-machine cooperation
Fig. 1. Concept of human-machine cooperation

From this viewpoint, several investigations of humanmachine cooperative systems have been reported. Murayama et al. developed the car window installation assist $\operatorname{robot}^{(2)}$. This robot conducted the task of installing the window glass on the car body with one worker. This task was conducted by two workers before the use of this robot. Takesue et al. reported a human-robot system based on position-based impedance control ${ }^{(3)}$. This system achieved steady contact with the environment. Hamaguchi et al. developed the cooperative transporting control method for two 7-degree-offreedom (DOF) robots and one human ${ }^{(4)}$. Hirata et al. proposed a transportation method for multiple robot helpers in cooperation with one human ${ }^{(5)}$. However, many conventional researchers have utilized position-based compliance control developed for industrial robots. The reaction force response is measured by a force sensor or estimated by an observer. In the position-based compliance controller, the force response is transformed into the position dimension using the virtual compliance model. If unexpected contact between the system and a human or the environment occurs, the position error may be large. Then, excessive force may be generated, since the position-based compliance controller reduces this position error.

This paper proposes a force-based compliance controller with a force threshold. This controller achieves both task 
realization and adaptation to its environment. By adapting to the environment, force exceeding the force threshold is not generated, since the force threshold is implemented as the force limit. Therefore, the application of excessive force to the environment is prevented. On the other hand, force exceeding the force threshold is generated during task realization. This motion selection is conducted by comparing the force command and the force threshold.

In addition, this paper discusses the application of the proposed controller to the grasping/manipulating control method for the realization of a safe human-machine cooperative system. In the human-machine cooperative system, proposed controllers are implemented. This paper defines the humanmachine cooperative motion as consisting of the autonomous motion and the human-assisting motion. These motion priorities are determined with reference to the force thresholds. The high-priority motion is autonomously controlled. On the other hand, the low-priority motion is shifted to the humanassisting motion. Therefore, it is easy to design the motion priorities in the human-machine cooperative system.

This paper is organized as follows. Section 2 introduces the control method in modal space for the realization of the grasping/manipulating motion. In Sect. 3, the force-based compliance controller using the force threshold is proposed and a human-machine cooperative grasping/manipulating system is developed with the proposed controller. In Sect. 4, experimental results are shown to confirm the validity of the proposed method. Conclusions are provided in Sect. 5.

\section{Grasping/Manipulating Control Method}

This section presents the grasping/manipulating control method $^{(6)-(8)}$ : First, the system is controlled in modal space; then, a complicated motion is divided into simple motions such as a grasping motion and a manipulating motion.

For the transformation from motor space to modal space, several studies have been reported such as the DFT matrix ${ }^{(7)}$, the quarry matrix ${ }^{(8)}$, and the Hadamard matrix ${ }^{(9)}$. In this paper, the quarry matrix is used for this transformation. Then, the transformation from motor space to modal space is expressed.

$$
\begin{aligned}
\boldsymbol{X}_{n} & =\boldsymbol{Q}_{n} \boldsymbol{x}_{n} \ldots \ldots \ldots \ldots \ldots \ldots \ldots \ldots \ldots \ldots \ldots \ldots \ldots \ldots \ldots \ldots \ldots \ldots \ldots \ldots \ldots \ldots \ldots \ldots \ldots \ldots \ldots \ldots \ldots
\end{aligned}
$$

where subscript $n$ represents a natural number. $\boldsymbol{Q}_{n} \in \mathfrak{R}^{n \times n}$ means the $n$-th order quarry matrix. $\boldsymbol{X}_{n}=\left[X_{1}, X_{2}, \cdots, X_{n}\right]^{T}$ and $\boldsymbol{F}_{n}=\left[F_{1}, F_{2}, \cdots, F_{n}\right]^{T}$ represent the $n$-th order position vector and force vector in modal space. $\boldsymbol{x}_{n}=\left[x_{1}, x_{2}, \cdots, x_{n}\right]^{T}$ and $\boldsymbol{f}_{n}=\left[f_{1}, f_{2}, \cdots, f_{n}\right]^{T}$ are the $n$-th order position vector and force vector in motor space. The inverse transformation is also described.

$$
\begin{aligned}
\boldsymbol{x}_{n} & =\boldsymbol{Q}_{n}{ }^{-1} \boldsymbol{X}_{n} \ldots \ldots \ldots \ldots \ldots \ldots \ldots \ldots \ldots \ldots \ldots \ldots \ldots \ldots \ldots \ldots \ldots \ldots \ldots \ldots \ldots \ldots \ldots \ldots \ldots \ldots \ldots
\end{aligned}
$$

Figure 2 shows the block diagram for the control method in modal space. In this paper, a disturbance observer (DOB) is implemented for the realization of robust acceleration control $^{(10)}$. A reaction force observer (RFOB) is utilized for the estimation of the external force ${ }^{(1)}$. Each source of information in modal space is independent. Therefore, it is easy

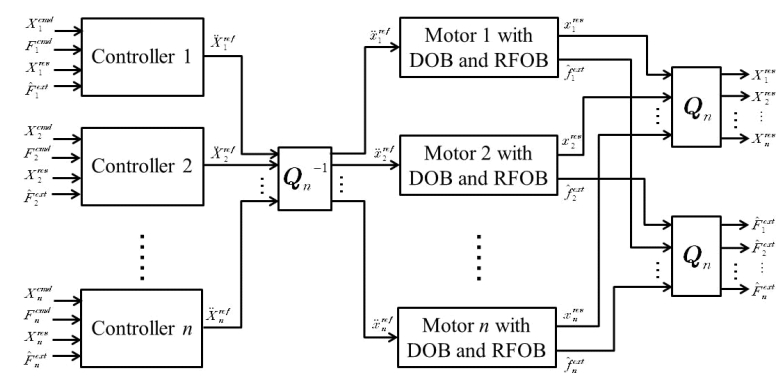

Fig. 2. Block diagram for control method in modal space

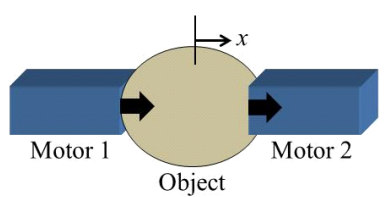

(a) Manipulating mode

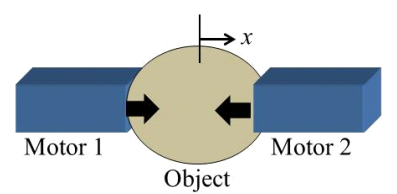

(b) Grasping mode

Fig. 3. Physical meaning in modal space (2 DOF)

to achieve the complicated motion by the implementation of several controllers. The second order quarry matrix and the third order quarry matrix are expressed.

$$
\begin{aligned}
\boldsymbol{Q}_{2} & =\frac{1}{2}\left[\begin{array}{cc}
1 & 1 \\
1 & -1
\end{array}\right] \ldots \ldots . \\
\boldsymbol{Q}_{3} & =\frac{1}{3}\left[\begin{array}{ccc}
1 & 1 & 1 \\
0 & 1 & -1 \\
2 & -1 & -1
\end{array}\right] \ldots
\end{aligned}
$$

For example, when the system has two DOFs, $\boldsymbol{Q}_{2}$ is applied for the transformation described in (1) and (2).

$$
\begin{aligned}
& {\left[\begin{array}{l}
X_{1} \\
X_{2}
\end{array}\right]=\frac{1}{2}\left[\begin{array}{cc}
1 & 1 \\
1 & -1
\end{array}\right]\left[\begin{array}{l}
x_{1} \\
x_{2}
\end{array}\right] .} \\
& {\left[\begin{array}{l}
F_{1} \\
F_{2}
\end{array}\right]=\frac{1}{2}\left[\begin{array}{cc}
1 & 1 \\
1 & -1
\end{array}\right]\left[\begin{array}{l}
f_{1} \\
f_{2}
\end{array}\right],}
\end{aligned}
$$

where $X_{1}$ and $F_{1}$ represent the manipulating (translation) modes along the $x$-axis related to the position and the force. $X_{2}$ and $F_{2}$ are the grasping modes related to the position and the force. Figure 3 shows the physical meaning in the twoDOF system. The origin of the coordinate system is set at the center of the initial object position. The direction of the $x$-axis is along the direction of movement of motor 1 . The position controller in the manipulating mode, and the force controller in the grasping mode, were implemented in the conventional researches. When the system has three DOFs, the manipulating (translation) mode along the $x$-axis, the manipulating (rotation) mode around the origin, and the grasping mode are extracted as shown in Fig. 4. References ${ }^{(6)-(8)}$ explain the details of the control method in modal space.

\section{Proposed Method}

This section proposes the force-based compliance controller with a force threshold and describes the development of a human-machine cooperative grasping/manipulating system using this proposed controller.

\subsection{Force-based Compliance Controller with Force} Threshold This subsection proposes the force-based compliance controller with the force threshold. When the 


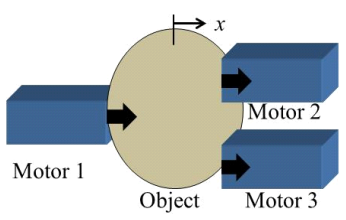

(a) Manipulating mode (translation)

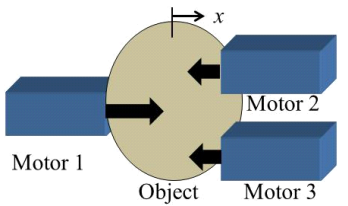

(c) Grasping mode

Fig. 4. Physical meaning in modal space (3 DOF)

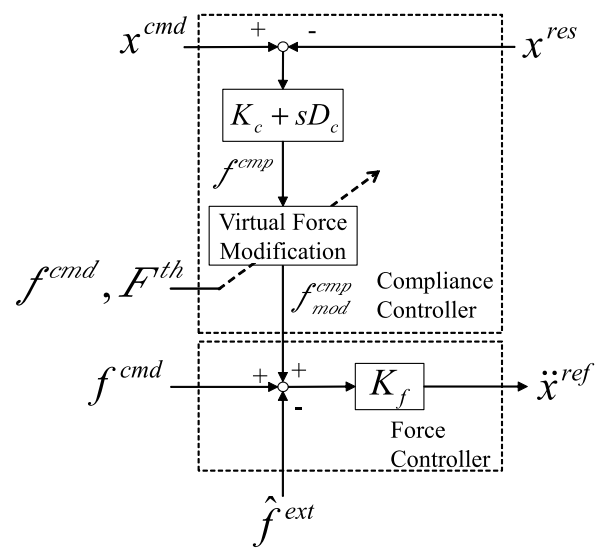

Fig. 5. Block diagram for force-based compliance controller with force threshold

system is in contact with the environment, the position is determined from the external force and environmental parameters such as the impedance, the mass, and the friction. Therefore, the proposed controller actualizes the force command during the pushing motion. On the other hand, position tracking is conducted when the system is removed from the environment since there is no external force from the environment. In addition, adaptation to the environment is actualized at the moment of the unexpected contact. The proposed controller can achieve the flexible motion depending on the contact condition.

Figure 5 shows the block diagram for the proposed forcebased compliance controller with the force threshold. The acceleration reference $\ddot{x}^{\text {ref }}$ is calculated as (9).

$$
\ddot{x}^{r e f}=K_{f}\left(f^{c m d}-\hat{f}^{e x t}+f_{m o d}^{c m p}\right), \cdot
$$

where $f^{c m d}, \hat{f}^{e x t}$ and $f_{\text {mod }}^{c m p}$ represent the force command, the reaction force added to the motor, and the modified virtual force, respectively. Superscript ref, cmd and ext mean the reference value, the command value, and the value from the external environment, respectively. ^ and $K_{f}$ represent the estimation value and the force gain.

The modified virtual force, $f_{m o d}^{c m p}$, is important for the flexible motion depending on the contact condition. $f_{\text {mod }}^{c m p}$ is obtained by two steps. In the first step, the virtual force $f^{c m p}$ is calculated from the position and velocity errors.

$$
f^{c m p}=K_{c}\left(x^{c m d}-x^{r e s}\right)+D_{c}\left(\dot{x}^{c m d}-\dot{x}^{r e s}\right), \cdots \cdots \cdots
$$

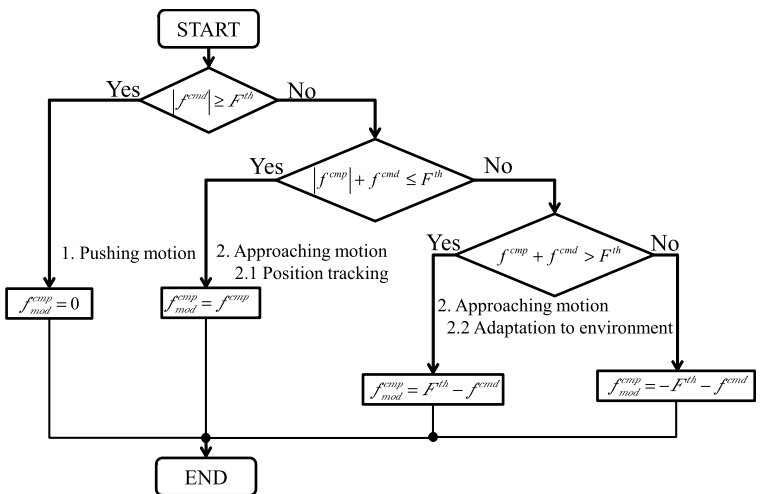

Fig. 6. Flow chart of virtual force modification

where $x$ and $\dot{x}$ represent the position and the velocity. The superscript res means the response value. $K_{c}$ and $D_{c}$ represent the virtual spring coefficient and the virtual damper coefficient. By using (10), the position and velocity errors are transformed into the virtual force.

In the second step, the virtual force is modified. $f_{m o d}^{c m p}$ is calculated from the relation of $f^{c m d}, f^{c m p}$ and the force threshold $F^{\text {th }}$. The proposed controller assumes the following motions:

1. Pushing motion $\left(\left|f^{\text {cmd }}\right| \geq F^{\text {th }}\right)$

2. Approaching motion $\left(\left|f^{c m d}\right|<F^{\text {th }}\right)$

2.1 Position tracking $\left(\left|f^{c m p}\right|+f^{c m d} \leq F^{t h}\right)$

2.2 Adaptation to environment

$$
\left(\left|f^{c m p}\right|+f^{c m d}>F^{t h}\right)
$$

Figure 6 shows the flow chart of the virtual force modification. In Fig. 6, the motion is judged to "Pushing motion" or "Approaching motion" at first.

$$
f_{\text {mod }}^{c m p}= \begin{cases}0 & :\left|f^{c m d}\right| \geq F^{t h} \\ f^{*} & :\left|f^{c m d}\right|<F^{t h}\end{cases}
$$

When the motion is treated as "Pushing motion" $\left(\left|f^{c m d}\right| \geq\right.$ $\left.F^{t h}\right)$, the virtual force is set to zero. Then, (9) is shifted to the force controller and the force command is realized. In "Pushing motion", force exceeding the force threshold is generated. On the other hand, $f^{*}$ is calculated as "Approaching motion" $\left(\left|f^{c m d}\right|<F^{\text {th }}\right)$.

$$
f^{*}= \begin{cases}F^{t h}-f^{c m d} & : f^{c m p}+f^{c m d}>F^{t h} \\ f^{c m p} & :\left|f^{c m p}\right|+f^{c m d} \leq F^{t h} \\ -F^{t h}-f^{c m d} & : f^{c m p}+f^{c m d}<-F^{t h}\end{cases}
$$

While $\left|f^{c m p}\right|+f^{c m d} \leq F^{\text {th }}$, the virtual force is not modified, and the position command is tracked.

On the other hand, the virtual force is modified while $\left|f^{c m p}\right|+f^{c m d}>F^{t h}$. In this case, the value of $f^{c m p}$ is large. This fact shows the position error occurs because of the unexpected contact. Therefore, the virtual force is modified for adaptation to the environment. In the case of $f^{c m p}+f^{c m d}>F^{t h}$, the acceleration reference is calculated using (9) and (12).

$$
\ddot{x}^{r e f}=K_{f}\left(F^{t h}-\hat{f}^{e x t}\right) \cdot
$$

Equation (13) is treated that the force command is equal to $F^{\text {th }}$ in the force controller. In this case, the force threshold is implemented as the force limitation. Therefore, force exceeding $F^{\text {th }}$ is not generated. From this viewpoint, the proposed 
controller conducts the safe motion.

The stability and performance analyses of the proposed controller are almost the same as these on the force-based variable compliance controller. These analyses are elaborated in a previous study ${ }^{(12)}$.

3.2 Human-machine Cooperative Grasping/Manipulating System This subsection describes the humanmachine cooperative grasping/manipulating system developed with the proposed controller.

3.2.1 Concept The proposed controller is applied to the grasping/manipulating control method. Proposed controllers are implemented from "Controller 1" to "Controller $n$ " as shown in Fig. 2. In other words, the proposed controller is utilized in each modal space. The proposed controller is set to the force threshold in each mode. The humanmachine cooperative motion consists of the autonomous motion and the human-assisting motion. By designing these force thresholds, it is possible to design the priorities of the autonomous motion and the human-assisting motion.

Considering the grasping/manipulating modes, the priority of the grasping mode should be the highest, since the manipulating motions cannot be realized without the grasping motion. On the other hand, when there are several manipulating modes $(n>2)$, the autonomous motion or the humanassisting motion is selected according to the motion priorities. The high-priority motion is autonomously controlled. On the other hand, the low-priority motion is shifted to the human-assisting motion. Then, the manipulation force of the operator is almost equal to the force threshold.

As a result, the priorities of the human-machine cooperative motion are designed by the force thresholds. In addition, it is possible to change the motion priorities by the modifications of the force thresholds in real time.

3.2.2 Design Method of Force Threshold In the proposed system, the number of the force thresholds is the same as the number of modal space. The design method of these force thresholds is described.

The force controller should be implemented considering the grasping mode. As shown in Fig. 6, (9) is shifted to the force controller, when the force command is larger than the force threshold. Therefore, the force threshold of the grasping mode is set as:

$$
F_{\text {grasp }}^{\text {cmd }}>F_{\text {grasp }}^{\text {th }}
$$

where $F_{\text {grasp }}^{\text {cmd }}$ and $F_{\text {grasp }}^{\text {th }}$ represent the force command and the force threshold in the grasping mode.

On the other hand, the design method of manipulating modes is discussed. This paper focuses on the design method of the force thresholds, when the system has three DOFs. It is possible to use the same method to design these values in other cases. The reaction forces added to the motors are calculated from (4) and (6).

$$
\begin{aligned}
& \hat{f}_{1}^{e x t}=\hat{F}_{1}^{e x t}+\hat{F}_{3}^{e x t} \cdots \cdots \cdots \\
& \hat{f}_{2}^{e x t}=\hat{F}_{1}^{e x t}+\frac{2}{3} \hat{F}_{2}^{e x t}-\frac{1}{2} \hat{F}_{3}^{e x t} \cdots \\
& \hat{f}_{3}^{e x t}=\hat{F}_{1}^{e x t}-\frac{2}{3} \hat{F}_{2}^{e x t}-\frac{1}{2} \hat{F}_{3}^{e x t}
\end{aligned}
$$

The design method assumes that the stable grasping motion is achieved $\left(\hat{F}_{3}^{e x t}=F_{\text {grasp }}^{\text {cmd }}\right)$. In addition, it is assumed that the one manipulating mode is shifted to "Adaption to environment" and the other manipulating mode is treated as "Position tracking". Then, these two conditions are derived: Condition 1: $\hat{F}_{1}^{e x t}= \pm F_{1}^{\text {th }}, \hat{F}_{2}^{\text {ext }}=0, \hat{F}_{3}^{\text {ext }}=F_{\text {grasp }}^{\text {cmd }}$, Condition 2: $\hat{F}_{1}^{\text {ext }}=0, \hat{F}_{2}^{\text {ext }}= \pm F_{2}^{\text {th }}, \hat{F}_{3}^{\text {ext }}=F_{\text {grasp }}^{\text {cmd }}$.

For the realization of the grasping motion, it is necessary to satisfy $\hat{f}_{1}^{e x t}>0$ and $\hat{f}_{2}^{e x t}<0$. The region of $F_{1}^{\text {th }}$ is calculated from (15)-(17) and condition 1.

$$
0<F_{1}^{\text {th }}<\frac{1}{2} F_{\text {grasp }}^{\text {cmd }} .
$$

The region of $F_{2}^{t h}$ is also obtained in condition 2 .

$$
0<F_{2}^{\text {th }}<\frac{3}{4} F_{\text {grasp }}^{\text {cmd }} .
$$

One of the manipulating modes is set to (18) or (19). Then, this manipulating mode is treated as the human-assisting motion. The other manipulating mode is autonomous motion.

\section{Experiments}

This section presents the experimental results that confirm the validity of the proposed method.

4.1 Experimental Setup Table 1 shows the control parameters used for the experiment. For the evaluation of the basic performance in the proposed system, this paper uses linear motors as one of the simpler systems available for achieving the grasping/manipulating motion. This paper conducted two experiments.

Case 1: Two linear motors are utilized as shown in Fig. 7(a). This system includes a manipulating (translation) mode and a grasping mode. The hard rubber block is treated as the object. The experiment in Case 1 consists of three steps implemented for ranges of time, $t$. In Step $1(0 \leq t<2)$, the

Table 1. Control parameters

\begin{tabular}{|l||l|l|}
\hline$m$ & Mass (Linear Motor) & $0.5 \mathrm{~kg}$ \\
$K_{f}$ & Force Gain & 1.0 \\
$K_{p}$ & Position Gain & 4900 \\
$K_{v}$ & Velocity Gain & 140 \\
$K_{c}$ & Virtual Spring Coefficient & $3600 \mathrm{~N} / \mathrm{m}$ \\
$D_{c}$ & Virtual Damper Coefficient & $120 \mathrm{Ns} / \mathrm{m}$ \\
$M_{c}$ & Virtual Mass Coefficient & $1.0 \mathrm{~kg}$ \\
$g_{\text {dis }}$ & Cut-off Frequency of DOB & $800 \mathrm{rad} / \mathrm{s}$ \\
$g_{\text {reac }}$ & Cut-off Frequency of RFOB & $800 \mathrm{rad} / \mathrm{s}$ \\
$s t$ & Sampling Time & $0.0001 \mathrm{~s}$ \\
\hline
\end{tabular}

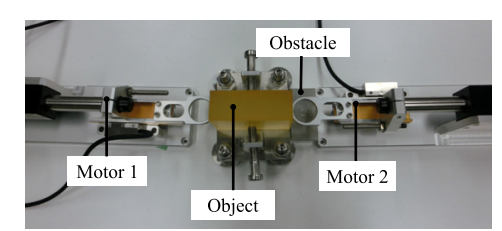

(a) Case 1

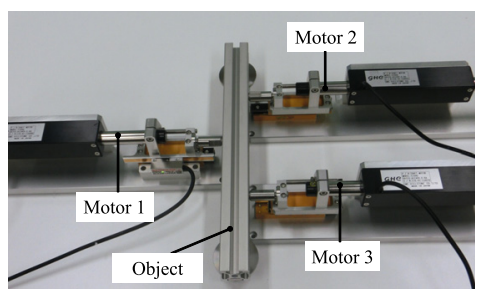

(b) Case 2

Fig. 7. Experimental setup 
system grasps the object. In Step $2(2 \leq t<6)$, the grasping/manipulating motion is implemented. In addition, there is the aluminum block as the obstacle. Therefore, the contact between the object and the obstacle occurs. In Step 3 $(6 \leq t<12)$, the operator directly manipulates the system to assist the manipulating mode. The position command of the manipulating mode in Step 2 and the force command of the grasping mode in Steps 1-3 are described as follows; $X_{1}^{\text {cmd }}=0.01 \sin (\pi t), F_{2}^{\text {cmd }}=2.5$. Other commands are set to $0\left(F_{1}^{c m d}=0.0, X_{2}^{c m d}=0.0\right)$. In addition, the experiment in Case 1 consists of two parts for the comparison between the conventional method and the proposed method.

Case 1-1: The position-based compliance controller ${ }^{(13)}$ in the manipulating mode and the force controller in the grasping mode are used as the conventional controller.

$$
\begin{aligned}
& \ddot{X}_{1}^{\text {ref }}=C_{p}(s)\left(X_{1}^{c m d}-X_{1}^{\text {res }}-X_{1}^{c}\right)-\ddot{X}_{1}^{c} \ldots \ldots \ldots \ldots \\
& K_{f} \hat{F}_{1}^{e x t}=M_{c} \ddot{X}_{1}^{c}+D_{c} \dot{X}_{1}^{c}+K_{c} X_{1}^{c} \\
& \ddot{X}_{2}^{\text {ref }}=K_{f}\left(F_{2}^{c \text { cmd }}-\hat{F}_{2}^{e x t}\right),
\end{aligned}
$$

where $C_{p}(s)\left(=K_{p}+s K_{v}\right)$ represents the position controller. $K_{p}, K_{v}, s$, and $M_{c}$ mean the position gain, the velocity gain, Laplace operator, and the virtual mass coefficient, respectively.

Case 1-2: The force-based compliance controllers with the force threshold are used in all modes. The force thresholds are set to $0.5 \mathrm{~N}$ in the manipulating mode and $1.0 \mathrm{~N}$ in the grasping mode $\left(F_{1}^{\text {th }}=0.5, F_{2}^{\text {th }}=1.0\right)$.

Case 2: Three linear motors are utilized as shown in Fig. 7(b). This system includes a manipulating (translation) mode, a manipulating (rotation) mode, and a grasping mode. The aluminum frame is treated as the object. In this case, the force-based compliance controllers with the force thresholds are used in all modes. The experiment in Case 2 consists of five steps. Table 2 shows the motion classification in Case 2. In Step $1(0 \leq t<2)$, the system grasps the object. As shown in Table 2, the grasping mode is autonomous motion in Case 2. In Step $2(2 \leq t<6)$, the human-machine cooperative motion, which consists of the autonomous motion and the human-assisting motion, is conducted. As shown in Table 2, the manipulation motion (rotation) is assisted by the operator. On the other hand, the manipulation motion (translation) is autonomously controlled. In Step $3(6 \leq t<8)$, the motion priorities of the manipulating modes are changed by the force thresholds. In Step $4(8 \leq t<12)$, the manipulation motion (translation) is assisted by the operator, and the manipulation motion (rotation) is autonomously controlled. In Step $5(t \geq 12)$, the stop motion is conducted. The commands can be described with (23)-(28).

$$
X_{1}^{c m d}= \begin{cases}0.01 \sin (\pi t) & :(2 \leq t \leq 6) \\ X_{1}^{\text {res }}(12) & :(t>12) \\ 0 & : \text { Otherwise }\end{cases}
$$

Table 2. Motion classification in Case 2

\begin{tabular}{|l||c|c|c|c|c|}
\hline \multicolumn{1}{|c||}{} & \multicolumn{5}{c|}{ Step } \\
\cline { 2 - 6 } & 1 & 2 & 3 & 4 & 5 \\
\hline \hline Manipulating (translation) & $\mathbf{A}$ & $\mathbf{A}$ & $\mathbf{A}$ & $\mathbf{H}$ & $\mathbf{A}$ \\
\hline Manipulating (rotation) & $\mathbf{A}$ & $\mathbf{H}$ & $\mathbf{A}$ & $\mathbf{A}$ & $\mathbf{A}$ \\
\hline Grasping & $\mathbf{A}$ & $\mathbf{A}$ & $\mathbf{A}$ & $\mathbf{A}$ & $\mathbf{A}$ \\
\hline A:Autonomous motion, H:Human-assisting motion
\end{tabular}

$$
\begin{aligned}
& X_{2}^{\text {cmd }}= \begin{cases}0 & :(t<8) \\
X_{2}^{\text {res }}(8)+0.005 \sin (\pi t) & :(8 \leq t \leq 12) \\
X_{2}^{\text {res }}(12) & :(t>12)\end{cases} \\
& X_{3}^{\text {cmd }}=0.0 \\
& F_{1}^{c m d}=0.0 \\
& F_{2}^{\text {cnd }}=0.0 \\
& F_{3}^{\text {cmd }}=4.0 \text {, }
\end{aligned}
$$

where $X_{i}^{\text {res }}(T)$ means the $X_{i}^{\text {res }}$ at $T \mathrm{~s}$ and $i=1,2$. The force thresholds were expressed.

$$
\begin{aligned}
F_{1}^{t h} & = \begin{cases}1.5 & :(0 \leq t \leq 6) \\
1.5-0.5(t-6) & :(6<t \leq 8) \\
0.5 & :(t>8)\end{cases} \\
F_{2}^{t h} & = \begin{cases}0.5 & :(0 \leq t \leq 6) \\
0.5+0.5(t-6) & :(6<t \leq 8) \\
1.5 & :(t>8)\end{cases} \\
F_{3}^{t h} & =2.0 \ldots \ldots \ldots \ldots \ldots \ldots \ldots \ldots \ldots \ldots \ldots \ldots
\end{aligned}
$$

From the relation between (26)-(27) and (29)-(30), the manipulating (translation) mode and the manipulating (rotation) mode are selected for "Approaching motion" as shown in Fig. 6. Then, the position commands are tracked, if there is no unexpected contact. If the system makes contact with the operator, the motion is shifted to "Adaption to environment". In the grasping mode, the proposed controller is shifted to the force controller, because of $F_{3}^{c m d}>F_{t h}^{3}$.

4.2 Experimental Results Figures 8-9 and Fig. 12 show the experimental results in Case 1 and Case 2. (a) and (b) in Figs. 8-9 and Fig. 12 represent the position responses and the force responses.

In Case 1-1, two linear motors and the conventional controllers are used. In Step 1, the grasping motion is achieved
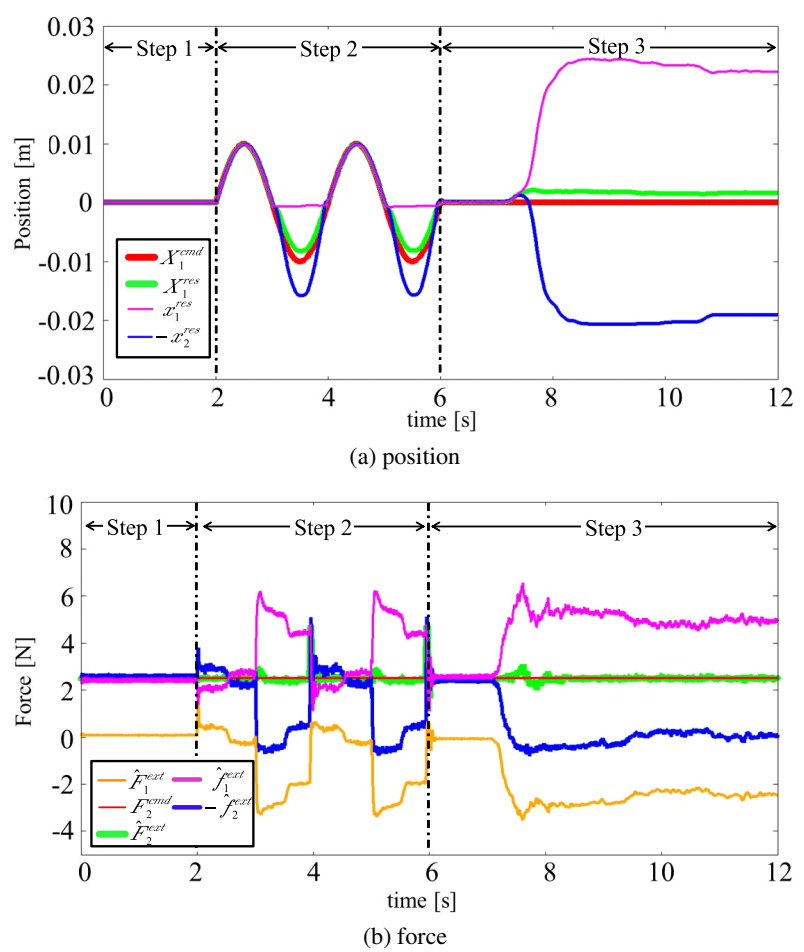

Fig. 8. Experimental results in Case 1-1 
as shown in Fig. 8(b). In Step 2, the grasping/manipulating motion is conducted. From Fig. 8(a), it can be seen that the manipulating (translation) mode response, $X_{1}^{\text {res }}$, is almost the same as the command, $X_{1}^{c m d}$, by the autonomous motion. However, the position error occurs from $3 \mathrm{~s}$ to $4 \mathrm{~s}$ and from $5 \mathrm{~s}$ to $6 \mathrm{~s}$, since the object is contact with the obstacle. At the moment of the contact between the obstacle and the object, the impact force is transmitted. This impact force is influenced by the force response in motor $1, \hat{f}_{1}^{\text {ext }}$, since motor 1 is in contact with the object. Therefore, the overshoot occurs in the force response of motor $1, \hat{f}_{1}^{e x t}$. On the other hand, the force response of motor $2, \hat{f}_{2}^{e x t}$, is equal to about $0 \mathrm{~N}$. This fact shows motor 2 is apart from the object. In other words, the grasping motion in Step 2 is not achieved. In Step 3, the operator assists the manipulating (translation) motion. Then, the grasping motion is not also actualized because of $\hat{f}_{2}^{e x t} \approx 0$. In addition, about $3 \mathrm{~N}$ is necessary to assist the manipulating (translation) motion, because of $\left|\hat{F}_{2}^{\text {ext }}\right|$.

In Case 1-2, the proposed controllers are used. In Step 1,
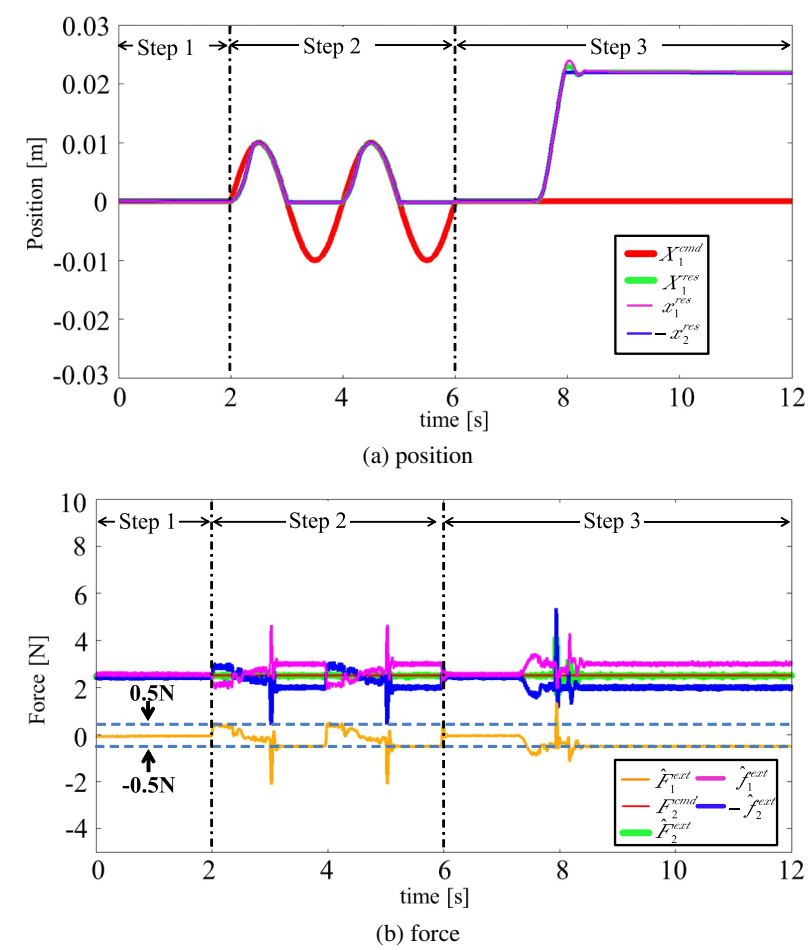

Fig. 9. Experimental results in Case 1-2
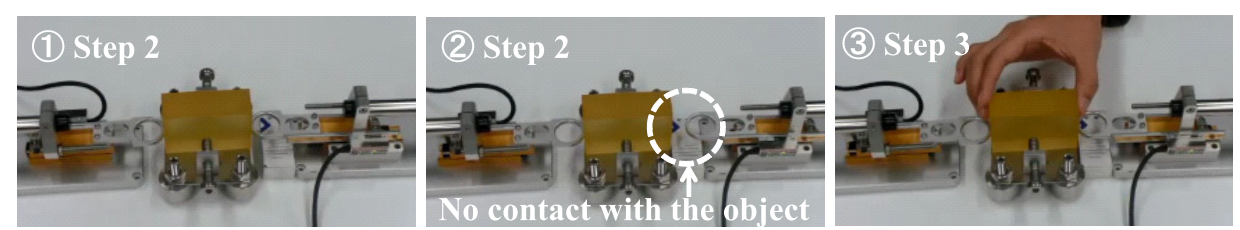

the grasping motion is also achieved as shown in Fig. 9(b). In Step 2, the grasping/manipulating motion is conducted. From Fig. 9(b), it can be seen that the manipulating (translation) mode response, $X_{1}^{r e s}$, is almost the same as for the command, $X_{1}^{c m d}$, from $2 \mathrm{~s}$ to $3 \mathrm{~s}$ and from $4 \mathrm{~s}$ to $5 \mathrm{~s}$. On the other hand, the position error occurs from $3 \mathrm{~s}$ to $4 \mathrm{~s}$ and from $5 \mathrm{~s}$ to $6 \mathrm{~s}$, because of the contact between the object and the obstacle. Therefore, the system stops at the obstacle position. Then, the force response of the manipulating mode, $\hat{F}_{2}^{e x t}$, is almost equal to the force threshold. From this point of view, the safe motion for the unexpected contact is actualized. In Step 3, the grasping motion is actualized as shown in Fig. 9(a). On the other hand, the manipulating (translation) motion is assisted by the operator. Then, the human-assisting motion is actualized by the little manipulation force, because of $\left|\hat{F}_{2}^{e x t}\right| \leq F_{2}^{t h}$.

Figures 10-11 show snapshots of the experiments for Case 1-1 and Case 1-2. By comparing Fig. 10 and Fig. 11, it can be seen that the main differences are shown in the second and fourth snapshots. In these snapshots of Fig. 10, the

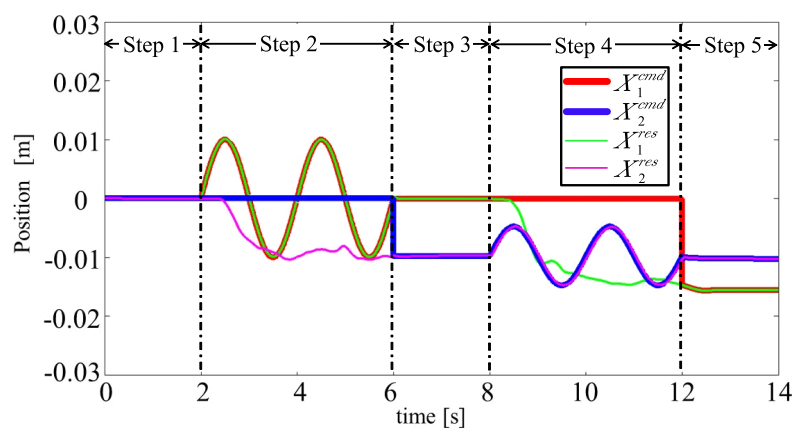

(a) position

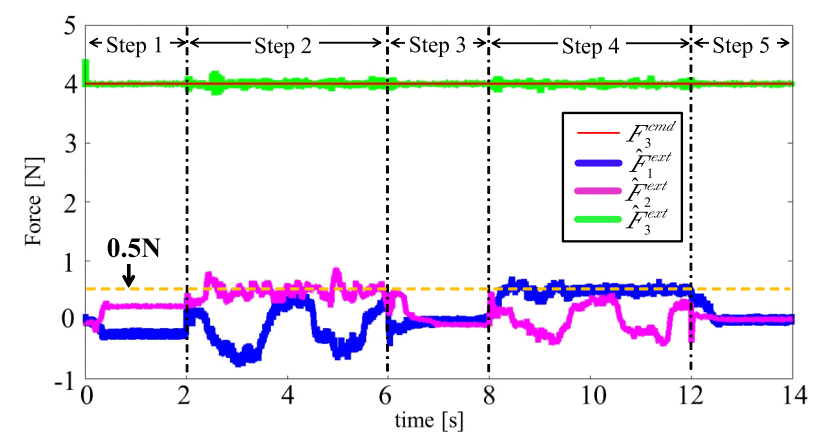

(b) force

Fig. 12. Experimental results in Case 2

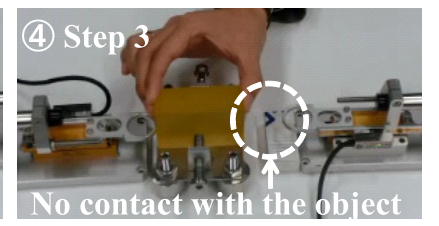

Fig. 10. Snapshots of experiment in Case 1-1
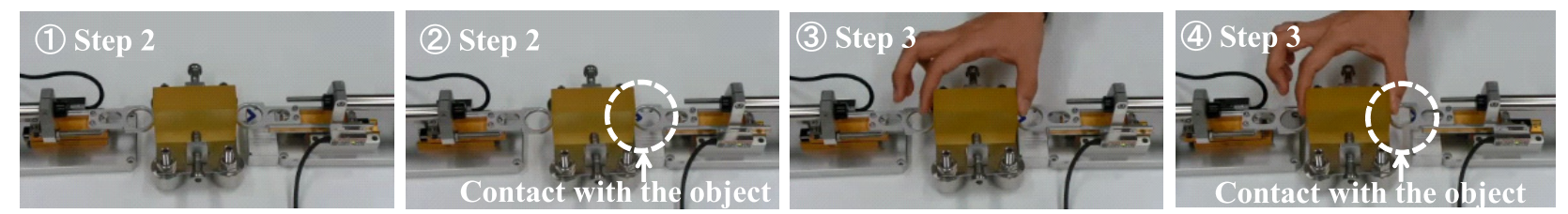

Fig. 11. Snapshots of experiment in Case 1-2 

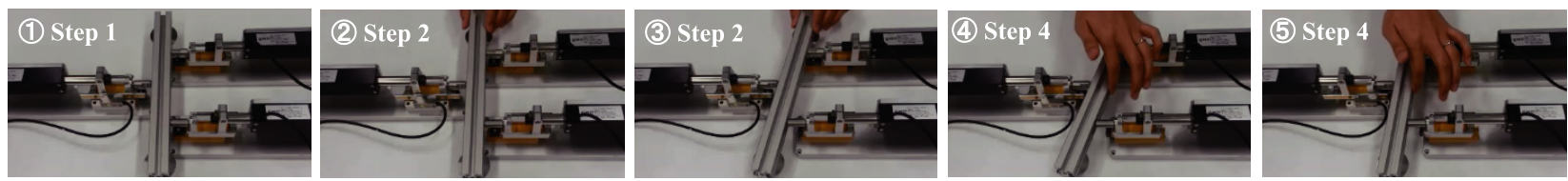

Fig. 13. Snapshots of experiment in Case 2

motor 2 is apart from the object. On the other hand, the motor 2 is always in contact with the object, as shown in Fig. 11. From these experimental results in Case 1, the force-based compliance controller achieves the flexible motion depending on the contact condition.

In Case 2, three linear motors and the proposed controllers are used. Therefore, the system has three modes: the manipulating (translation) mode, the manipulating (rotation) mode and the grasping mode. In Step 1, the system grasps the object as shown in Fig. 12(b). In Step 2, the priority of the manipulating (translation) mode is higher than one of the manipulating (rotation) mode, because of $F_{1}^{\text {th }}>F_{2}^{\text {th }}$. Therefore, the manipulating (translation) mode response, $X_{1}^{\text {res }}$, follows the command, $X_{1}^{c m d}$, as shown in Fig. 12(a). On the other hand, the manipulating (rotation) mode response, $X_{2}^{\text {res }}$, is assisted by the operator. From this point of view, the human-machine cooperative motion is achieved. In Step 3, the grasping motion is still being carried out. The motion priorities of the manipulating modes are changed as shown in (29) and (30). In Step 4, the manipulating (rotation) mode is autonomously controlled. The manipulating (translation) mode response, $X_{1}^{r e s}$, is assisted by the operator. This fact shows that the motion priorities of manipulating modes are changed in real time. In Step 5, the system stops during the grasping the object. In Case 2, $\left|\hat{F}_{1}^{\text {ext }}\right|$ and $\left|\hat{F}_{2}^{e x t}\right|$ are almost within the force thresholds. Therefore, the operator easily modifies the manipulating modes.

Figure 13 shows snapshots of the experiment for Case 2. In the first snapshot, the system grasps the object. In the second and third snapshots, the manipulating (translation) motion is autonomously controlled. On the other hand, the manipulating (rotation) motion is assisted by the operator. In the fourth and fifth snapshots, the manipulating (translation) motion is assisted by the operator, and the manipulating (rotation) motion is autonomously controlled. From these experimental results in Case 2, the validity of the proposed method is confirmed.

\section{Conclusions}

This paper describes a proposed force-based compliance controller with a force threshold. This controller achieves both task realization and adaptation to the environment. In "Adaptation to environment", force exceeding the force threshold is not generated. On the other hand, force exceeding the force threshold is generated in "Pushing motion". Therefore, it is possible to achieve flexible and safe motion depending on the contact condition. In addition, the humanmachine cooperative grasping/manipulating system using the force-based compliance controller was developed. The priorities of the human-machine cooperative motion are easily designed by the force thresholds. Then, the autonomous motion and the human-assisting motion are actualized at the same time.
This paper summarizes tests conducted in two experiments. In Case 1, the comparison between the conventional controller and the proposed controller was shown. In Case 2, the autonomous motion and the human-assisting motion were achieved at the same time. From these experimental results, the validity of the proposed method was confirmed. For future work, we propose to apply the method to several mobile systems as shown in Fig. 1(b).

\section{Acknowledgment}

This research was partially supported by Japan Society for the Promotion of Science under Grant-in-Aid for Scientific Research (S), 25220903.

\section{References}

( 1 ) International Organization for Standardization: http://www.iso.org/iso/home. html

( 2 ) H. Murayama, N. Takesue, K. Matsumoto, H. Konosu, and H. Fujimoto: "Development of a Car Window Installation Assist Robot", Journal of the Robotics Society of Japan, Vol.28, No.5, pp.624-630 (2010) (in Japanese)

( 3 ) N. Takesue, S. Nobata, and H. Fujimoto: "Admittance Control Method Achieving Steady Contact", Journal of the Robotics Society of Japan, Vol.26, No.6, pp.635-642 (2008) (in Japanese)

( 4 ) M. Hamaguchi and T. Taniguchi: "Cooperative Transporting Control for Two Redundant Manipulators and One Human (A Method of Obstacle Avoidance Utilizing Kinematic Redundancy)", Transactions of the JSME Series $C$, Vol.79, No.808, pp.4734-4757 (2013) (in Japanese)

( 5 ) Y. Hirata, T. Hatsukari, K. Kosuge, H. Asama, H. Kaetsu, and K. Kawabata: "Transportation of an Object by Multiple Distributed Robot Helpers in Cooperation with a Human", Transactions of the JSME Series C, Vol.68, No.668, pp.181-188 (2002) (in Japanese)

( 6 ) T. Tsuji, K. Ohnishi, and A. Šabanovic̀: "A Controller Design Method Based on Functionality", IEEE Transactions on Industrial Electronics, Vol.54, No.6, pp.3335-3343 (2007)

( 7 ) R. Kubo and K. Ohnishi: "Mechanical recognition of unknown environment using active/passive contact motion", IEEE Transactions on Industrial Electronics, Vol.56, No.5, pp.1364-1374 (2009)

( 8 ) S. Katsura and K. Ohishi: "Quarry of Modal Information from Environment for Advanced Motion Control”, IEEJ Trans. IA Vol.126, No.4, pp.372-378 (2006)

( 9 ) M. Morisawa and K. Ohnishi: "Motion Control Taking Environmental Information into Account", EPE Journal, Vol.12, No.4, pp.37-41 (2002)

(10) K. Ohnishi, M. Shibata, and T. Murakami: "Motion Control for Advanced Mechatronics", IEEE/ASME Transactions on Mechatronics, Vol.1, No.1, pp.56-67 (1996)

(11) T. Murakami, F. Yu, and K. Ohnishi: "Torque Sensorless Control in Multidegree-of-Freedom Manipulator", IEEE Transactions on Industrial Elements, Vol.40, No.2, pp.259-265 (1993)

(12) N. Motoi, T. Shimono, R. Kubo, and A. Kawamura: "Task Realization by a Force-based Variable Compliance Controller for Flexible Motion Control Systems", IEEE Transaction on Industrial Electronics, Vol.61, No.2, pp.1009-1021 (2014)

(13) N. Nishikawa, Y. Fujimoto, T. Murakami, and K. Ohnishi: "Variable Compliance Controller for 3 Dimensional Biped Robot considering Environmental Fluctuations", IEEJ Trans. IA, Vol.119, No.12, pp.1507-1513 (2000) (in Japanese) 
Naoki Motoi (Member) received the B.E. degree in system design ค. engineering and the M.E. and Ph.D. degrees in integrated design engineering from Keio University, Japan, in 2005, 2007 and 2010, respectively. In 2007, he joined the Partner Robot Div., Toyota Motor Corporation, Japan. From 2011 to 2013, he was a research associate at Yokohama National University. Since 2014, he has been with Kobe University, Japan, where he is a currently lecturer. His research interests include robotics, motion control, and haptic.
Ryogo Kubo (Member) received the B.E. degree in system design

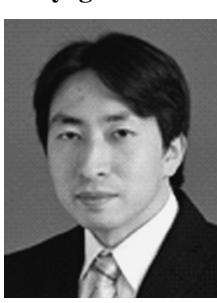
engineering and the M.E. and Ph.D. degrees in integrated design engineering from Keio University, Japan, in 2005, 2007 and 2009, respectively. In 2007, he joined the NTT Access Network Service Systems Laboratories, NTT Corporation, Japan. Since 2010, he has been with Keio University, Japan, where he is currently an assistant professor at the Department of Electronics and Electrical Engineering. His research interests include system control, optical communications, networking, sensor-actuator networks, smart grid and mechatronics. 